I. OVERVIEW OF FMS PROBLEMS AND SOLUTION APPROACHES 



\title{
DESIGN, PLANNING, SCHEDULING, AND CONTROL PROBLEMS OF FLEXIBLE MANUFACTURING SYSTEMS
}

\author{
K.E. STECKE \\ Graduate School of Business Administration, The University of Michigan, \\ Ann Arbor, Michigan 48109-1234, U.S.A.
}

\begin{abstract}
The design and use of flexible manufacturing systems (FMSs) involve some intricate operations research problems. FMS design problems include, for example, determining the appropriate number of machine tools of each type, the capacity of the material handling system, and the size of buffers. FMS planning problems include the determination of which parts should be simultaneously machined, the optimal partition of machine tools into groups, allocations of pallets and fixtures to part types, and the assignment of operations and associated cutting tools among the limited-capacity tool magazines of the machine tools. FMS scheduling problems include determining the optimal input sequence of parts and an optimal sequence at each machine tool given the current part mix. FMS control problems are those concerned with, for example, monitoring the system to be sure that requirements and due dates are being met and that unreliability problems are taken care of. This paper defines and describes these FMS problems in detail for OR/MS researchers to work on.
\end{abstract}

\section{Keywords and phrases}

FMS design problems, FMS planning problems, FMS scheduling problems, FMS control problems.

\section{Introduction}

Flexible manufacturing systems (FMSs), being somewhat similar and yet different from conventional manufacturing systems, provide new and different problems for the OR/MS community to solve. In this paper, short descriptions are provided of the various FMS management problems that need to be addressed at different stages of an FMS's life cycle, from conception through to implementation and operation. Some of these problems have been examined to some extent from varying points of view and using different $O R$ models and various solution techniques. 
This paper describes the problems and decisions that have to be addressed during the design, planning, scheduling and, finally, the actual control of an FMS. A companion paper [6] provides some information on models that can and have been used to evaluate some of these decisions.

An initial issue that has to be addressed is whether or not flexible manufacturing is really applicable to the proposed application. There are technological considerations that affect this decision. Also, often, some sort of cost justification is attempted, but this problem is difficult and the numbers can be manipulated to justify any decision. What is also important are the strategic abilities that flexible manufacturing provides. It is very difficult to quantify the abilities to respond to the demand for the FMS products, or to quickly introduce new part numbers. Nevertheless, cost justification is required and some of the papers in this volume do address this issue in an appropriate manner.

When the management decision has been made that flexible manufacturing is the way the company will go for production in a particular department, then all of the following problems and issues have to be addressed.

\section{FMS design problems}

In developing an FMS design, there is a partial ordering to some of the decisions that have to be made. Some decisions must precede others in time. We partition these into initial specification decisions and subsequent implementation decisions. These decisions are now defined and described further if additional explanation is required.

\subsection{INITIAL SPECIFICATION DECISIONS}

First, the manufacturing requirements need to be specified.

(1) Determine the range or families of components or part types to be produced.

From all of the part types which the factory produces, identify a subset to be manufactured and/or assembled on the FMS.

This is only an initial specification of what it is desired to manufacture on the system. This will change in time. Some of the benefits of flexible manufacturing includes the ability to respond to changing markets, and to quickly and efficiently incorporate design or process changes, or to use new materials. New part numbers can be added in the future.

From this broad idea of what the FMS will manufacture, the capacity and functional requirements can be specified, in terms of machine time and cutting tools required. In particular: 
(2) Determine how these part types shall be manufactured. This consideration eventually specifies the numbers and types of machine tools and robots that are required.

First, a process plan has to be determined for each part type. Each process plan defines not only which type (or sometimes, types) of machine tool shall perform each operation, but also determines the appropriate cutting tools and cutting conditions (i.e. the speed and feed rate of each cutting tool as well as the depth of the cut). These are required to determine the processing time of each operation in the process plan. With this information, the capacity required (both tool magazine capacity and processing time capacity), in terms of the number of machine tools of each type, can be determined. A process plan also provides a partial precedence ordering among the operations.

(3) Specify what types of different flexibilities are required or desired and the amounts of each.

Browne et al. [1] defined and described eight types of flexibilities that all FMSs theoretically can have. Buzacott [2] begins to quantify some of these flexibilities. All FMSs have varying amounts of each. In addition, no FMS utilizes all of its potential flexibility. This is because, in general, 'more flexibility' will be both more expensive and more difficult to utilize or to take advantage of. For example, flexible routing is more difficult to manage than fixed routing. Also, real-time control of the flow of parts through the system (where a decision on an act is made whenever it is near the time for some decision or action to occur, and is based on the particular state of the FMS near the time of that action) is much more difficult to implement than a fixed, static schedule of work flow.

(4) Determine the type of FMS that shall be developed.

In Browne et al. [1], the different types of flexibilities are used to classify different FMS types (according to how 'flexible' an FMS is). Of course, these FMS types range from somewhat inflexible (perhaps a flexible transfer line having a fixed process flow) to very flexible FMSs, having a widely varying process flow, even for parts of identical part type. This decision will help specify the amount of automation that will be included in the system and the type of control strategies for the different system components. 
(5) Specify the type, then capacity, of the material handling system.

The parts can be automatically transported throughout the FMS via roller conveyors, two-way tow-line tracks, or wire-guided carts, for example. In the latter two cases, the number of carts has to be determined. Stecke and Browne [5] further sub-classify various types of FMSs according to their methods of handling materials.

(6) First the type, and then the size, of the buffers has to be specified.

The buffer provides a queueing place for in-process inventory. There can be a central buffer area or a small, local buffer at each machine tool or both or neither. Some systems provide no area for a buffer and in-process inventory remains on the material handling system. Whether storage is centralized or local, the buffer size has to be determined. The trade-offs involve, in part, the space and the cost of the buffers (too much space) versus having enough buffers to help keep the machine tools utilized.

(7) The hierarchy among the computers controlling the different aspects of production has to be specified.

This control structure also specifies which computers communicate with which (their interconnections) and at what levels of the control. Data transmission devices are specified. There may be a computer controlling the machining operations of the machine tools, which determines and then downloads the appropriate part program that describes each cutting operation to the machine tool. There may be another computer controlling the material handling system. There may be still another supervisory computer controlling these computers. The structure may be $\mathrm{CNC}$ and/or DNC, for example.

(8) The vendors have to be chosen.

\subsection{SUBSEQUENT IMPLEMENTATION DECISIONS}

(9) The layout of the FMS has to be determined.

The layout, in conjunction with the type of material handling system, determines the travel time from machine tool to machine tool and impacts the total system travel time. Travel time and distance information is useful to help determine appropriate scheduling strategies. 
(10) The number of pallets has to be determined.

The number of pallets specifies the maximum number of parts that will be in the system. Determining the appropriate number of pallets is important because having too few pallets available causes system underutilization; having too many pallets causes system congestion and is also overly expensive.

(11) The number and design of the fixtures of each fixture type has to be determined.

Having too few of a particular fixture type causes unnecessary part waiting time; having too many fixtures results in unnecessary expense and wasted space/storage.

(12) The strategies for running the FMS have to be specified.

Overall, general planning and control objectives are needed in order to check that the FMS's physical design is feasible and will perform as expected. Detailed planning, loading, and scheduling algorithms will need to be developed later.

(13) The software development tasks need to be specified and implemented, perhaps within a project management setting.

These tasks include writing software for the control structure, tool management, inventory control, and scheduling, and then the implementation of each.

These design problems have been defined and presented here in a sequential manner. In fact, there is such a logical ordering, a sequencing to these design decisions that have to be made. However, many of the problems are interrelated. Some problems can be solved simultaneously. There will be a great deal of iteration back and forth among candidate solutions before the best FMS design is determined.

Once the system is designed, implemented, and in production, the management concern now turns to determining how to best run the system.

\section{FMS planning problems}

The production management problems associated with a manufacturing system can be viewed in a disaggregate manner in terms of the two distinct but intimately related functions of planning and scheduling. Now in an FMS, a part will not be routed 
to a particular machine tool of the correct type unless all of the cutting tools that are required for the part's next operation are already loaded into the machine tool's limited-capacity tool magazine. This indicates that some decisions have to be made even before production begins.

We define FMS planning problems to be those decisions that have to be made before the FMS can begin to produce parts. Once the FMS is 'set-up', production can start. The FMS scheduling problems are those concerned with running the system, of scheduling the flow parts throughout the FMS, and are the subject of sect. 3 .

At the FMS planning stage, the FMS has been implemented and is in production. From the part numbers which are processed on the system, there is a subset for which production orders exist, perhaps requirements from another department in the factory or from a sister plant or customer orders, or maybe forecasted demand. Then the FMS planning problems are as follows:

(1) From the list of part types for which production orders of various sizes are specified, choose a subset of part types for immediate and simultaneous manufacture.

This part type selection decision can be made in various ways. Due dates may be considered. Or part types may be chosen that are compatible in the sense that each type mainly utilizes a different machine type, and so, when machined simultaneously, they help attain a good, overall system utilization.

Once the part types have been selected, some aggregate information can be easily calculated concerning the total processing requirements and total number of tool slots required from all machine types.. Relating this information to the capacity available per machine of each type, both processing time and tool magazine capacity, can help address the next problem.

(2) Partition the machines of each type into machine groups.

Machines in a particular machine group are said to be pooled when they are identically tooled, and are each capable of performing the same operations during real time. Pooling machines can improve most system performance measures.

(3) Determine the production ratios at which the selected set of part types should be maintained at over time on the system.

(4) Allocate the limited number of pallets and the fixtures of each fixture type among the selected part types. 
The effect of allocating these limited resources is to revise the production ratios found in problem 3 .

(5) Allocate the operations and associated cutting tools of the selected part types among the (grouped) machines.

When the FMS planning problems have been solved, and all of the cutting tools have been loaded into the appropriate tool magazines, production can begin. These FMS planning problems can be solved sequentially, or iteratively, or several simultaneously. They can be re-solved as often as every couple of days or weeks. They may require re-solving if one of the machine tools is down for a long time. Whenever the production requirements for one of the selected part types are complete, this could trigger the solution of the planning problems again. For example, the cutting tools for the finished part type are no longer required, so either the reduced set of part types can be machined, possibly signaling a regrouping of machines to enable more pooling, or one or more part types can be added to the set of selected part types, signaling again a repartitioning of the machine tools into different groups.

\section{FMS scheduling problems}

FMS scheduling problems are concerned with running the FMS during real time once it has been set up during the planning stage which is in advance of actual production. There are many possible approaches that can be taken to schedule the manufacture of parts through the system. Different approaches might be applicable in different situations. Some of the problems inciude the following:

(1) Determine the optimal sequence at which the parts of the selected part types are to be input into the system.

Sometimes the part types have to be produced in certain relative ratios, say for assembly purposes. A periodic input sequence might also be appropriate for some types of FMSs. Producing to maintain certain production ratios of part types on the system may be appropriate. Also, a fixed, pre-determined input sequence may be appropriate. In other situations, a flexible, real-time decision concerning which part to input next may be best.

(2) Appropriate scheduling methods and algorithms have to be developed.

Scheduling aids can range from simple dispatching rules to complex algorithms or procedures incorporating look-ahead features. Most of the 
classical scheduling literature has been concerned with generating off-line schedules for a manager to try to use. In particular, a scheduling algorithm is often applied to some input data, resulting in a fixed schedule specifying which operations would be performed on which machine tools and when. More appropriate for an FMS might be a real-time, on-line scheduling policy, with scheduling decisions based on the actual state of the system (which machines up, which parts waiting for which machines, ... ). If the FMS were carefully set up during the planning stage, then a realtime scheduling function might be easier to apply. Perhaps more important during the planning stage, the due date criterion could also apply during the scheduling.

(3) If there are several parts waiting to be processed by the same machine tool, determine the priorities among these parts.

Random processing of parts in batches of size one is commonly referred to regarding FMS scheduling. However, a small amount of intelligent ordering of parts can greatly improve an FMS's performance. It may be appropriate to determine an optimal (dynamic) sequence at each machine tool. Perhaps this sequence could be periodic. Perhaps a simple (or complex) dispatching rule suffices.

Many of the usual performance measures are important, such as maximize production or machine utilization (the system is very expensive), or minimize inventory or flowtime, subject to meeting due dates, in systems in which the due date criteria are relevant.

\section{FMS control problems}

We define FMS control problems to be those associated with the continuous monitoring of the system, the keeping track of production to be certain that production requirements and due dates are being met as scheduled.

(1) Determine a policy to handle machine tool and other breakdowns. (The policy should be determined during the design phase. It is implemented during the control of an FMS.)

If one or more machine tools are down for a long period of time, should the schedule be revised; or should some procedures be developed that will force production to return to the original, planned schedule as soon as possible; or should a new schedule be developed? It should be noted 
that pooling machines into groups during the planning stage, in conjunction with real-time scheduling, allows machine tool breakdowns to be automatically handled. Also, a related problem is the following: if several breakdowns need attention, what should the maintenance person address his or her attention to next?

(2) Determine scheduled, periodic, preventive maintenance policies.

Preventive maintenance can be scheduled along with the production requirements as part of the weekly schedule, or it can be planned for to occur on an off shift or a weekend.

(3) Determine in-process and/or finished goods inspection policies.

The points of inspection of part dimensions as well as the frequency of each inspection have to be determined. Inspection equipment has to be selected and implemented.

(4) Procedures for tool life and process monitoring and data collection, as well as for updating the estimates of tool life, have to be specified.

\section{Solution aids}

There are many models available that can be applied to help answer some of the preceding questions. Each model can structure the problems differently. Each model ignores or aggregates some features of the system to focus on particular aspects. The models have provided either operational or qualitative insights into some of the FMS decision problems.

A list of several of the models that can and have been applied include: simulation, group technology, computer aided process planning, queueing networks, mathematical programming (linear, nonlinear, integer), perturbation analysis, Petri nets, and artificial intelligence. Buzacott and Yao [3] review analytic models, in particular queueing networks. Suri [6] overviews some of these models in a companion paper in this volume. Stecke [4] also describes the use of these models.

Many researchers have used different sets of these models to address many of the FMS problems overviewed here. Since most of the relevant literature references are noted in the various papers in this volume, we do not provide an extensive bibliography here. 


\section{References}

[1] J. Browne, D. Dubois, K. Rathmill, S.P. Sethi and K.E. Stecke, Classification of flexible manufacturing systems, The FMS Magazine 2, No. 2(1984)114.

[2] J.A. Buzacott, The fundamental principles of flexibility in manufacturing systems, Proc. 1st Int. Conf. on Flexible Manufacturing Systems, Brighton, U.K., 1982.

[3] J.A. Buzacott and D.D.W. Yao, Flexible manufacturing systems: A review of models, Working Paper No. 7, University of Toronto, Ontario, Canada, 1982.

[4] K.E. Stecke, Useful models to address FMS operating problems, Proc. IFIP Conference, Advances in Production Management Systems, Budapest, Hungary, 1985.

[5] K.E. Stecke and J. Browne, Variations in flexible manufacturing systems according to the relevant types of automated materials handling, Material Flow 2, Nos. 2/3(1985) 179.

[6] R. Suri, An overview of evaluative models for flexible manufacturing systems, Proc. 1st ORSA/TIMS Special Interest Conf. on Flexible Manufacturing Systems: Operations Research Models and Applications, Ann Arbor, Michigan (1984) p. 8. 